\title{
Las Comisiones como órganos de relación entre Gobierno Vasco y Diputaciones Forales
}

\section{Ainhoa Novo Arbona *}

\section{Introducción}

La doble diferenciación funcional y territorial del poder genera sistemas de decisión en los que las responsabilidades gubernamentales aparecen desagregadas en una serie de subsistemas dotados de funciones, competencias y recursos limitados. La distribución de competencias y funciones entre los diferentes niveles territoriales de gobierno (Estado, Comunidades Autónomas, Provincias y Ayuntamientos) hacen necesaria la existencia de órganos de interrelación para la gestión de las mismas. En el caso español, la relación entre las Comunidades Autónomas y el Estado se lleva a cabo a través de tres tipos de órganos: 1. Órganos de colaboración que responden a las competencias estatales de coordinación; 2. Órganos de colaboración que dan participación a las Comunidades Autónomas en el ejercicio de competencias estatales y que son a la vez órganos de coordinación de los diferentes departamentos ministeriales del Estado, y 3. Las Conferencias Sectoriales (ToRnos, 1994: 85-87).

Los primeros, los órganos de colaboración que responden a las competencias estatales de coordinación, son: el Consejo de Política Fiscal y Financiera, el Consejo Interterritorial del Servicio Nacional de Salud o el Consejo General de la Ciencia y la Tecnología. Los segundos, los órganos de colaboración que dan participación a las Comunidades Autónomas en el ejercicio de competencias estatales y que son a la vez órganos de coordinación de los diferentes departamentos ministeriales del Estado, son: la Comisión de Protección Civil, el Consejo Superior de Política de Seguridad, el Consejo Superior de Tráfico o la Comisión para la Competitividad Industrial. Las terceras, y más conocidas, son las Conferencias Sectoriales, como pueden ser: la Conferencia Sectorial del Plan Nacional sobre Drogas, la Conferencia Sectorial de Carreteras o la Conferencia sectorial de la Vivienda (Tornos, 1994: 85-87).

En la Comunidad Autónoma del País Vasco (en adelante, CAPV), el poder se divide de forma funcional, pero también territorial. Así, además de la división competencial entre el Estado y la CAPV, existe un reparto de materias también dentro de la CAPV entre sus diferentes gobiernos territoriales, como son los Territorios Históricos y los Municipios. Esto genera un sistema de interdependencia, de tal forma que las decisiones que se adoptan en cualquiera de los niveles territoriales de poder acaban afectando de forma directa o indirecta a otra institución. Por tanto, en la CAPV, también se hace necesario la existencia de órganos de participación, colaboración o coordinación.

Al igual que ocurre a nivel estatal, dentro de la CAPV podemos encontrar diferentes tipos de órganos que tienen como objeto el apoyo para el funcionamiento de un sistema institucional competencial y funcionalmente descentralizado. Tal y como se podrá comprobar en el apartado siguiente, esta investigación se dirige a estudiar un tipo de órgano determinado y no todos los existentes, sin olvidar, sin embargo, la importancia de todos ellos, en general, para el buen funcionamiento de las instituciones.

Los órganos interinstitucionales han sido importantes en todos los sistemas descentralizados, pero hoy en día su importancia ha ido incrementándose debido, por un lado, a la existencia de una realidad cada vez más cambiante $y$, por otro, a la intervención de la Administración pública en nuevas áreas. 
A todo ello hay que añadir que, tal y como ocurre a nivel estatal, el sistema institucional implantado en la CAPV es de reciente creación, con apenas dos décadas de tradición, por lo que todavía tiene problemas de definición de límites de actuación y de funcionamiento.

La CAPV además cuenta con un problema añadido: el desacuerdo político sobre cuál debe ser el sistema institucional vasco, lo que ha llevado a que se cree un sistema institucional de «petachos», basado en un la ley ${ }^{1}$ ambigua con poco apoyo parlamentario y acuerdos puntuales. Esta indefinición del papel de las diferentes instituciones dentro del sistema institucional vasco hace imprescindibles instrumentos de coordinación entre las diferentes instituciones. Así lo señala el informe sobre el marco institucional interno elaborado en $1989^{2}$, en el que, además de reflejar los problemas que la aplicación de la Ley de Relaciones entre las Instituciones Comunes de la Comunidad Autónoma y los Órganos Forales de sus Territorios Históricos (más conocida como Ley de Territorios Históricos, en adelante LTH), ha provocado, afirma, la necesidad de órganos interinstitucionales de coordinación. Es en este marco donde debe entenderse la presente investigación.

El artículo se centra en el estudio de los órganos creados por ley o por decreto, por los diferentes departamentos del Gobierno Vasco en los que participan las Diputaciones Forales en la quinta legislatura del Gobierno Vasco, que va desde 1994 a 1998. A pesar de que estos órganos reciben diferentes denominaciones, nos inclinaremos por considerar como comisiones a todos. Quedan fuera de análisis aquellos órganos cuya creación viene obligada por alguna de las leyes orgánicas o no vertebradoras del sistema institucional de la CAPV, como son: e] Estatuto de Autonomía del País Vasco ${ }^{3}$ (en adelante, EAPV), la LTH $^{4}$ y la Ley de Concierto Económico (en adelante, LCE) ${ }^{5}$ y cuya existencia no depende de la voluntad unilateral del Gobierno Vasco. Por ejemplo: la Comisión Arbitral (art. 39 del EAPV), la Comisión Mixta (art. 41.2.e) del EAPV y art. 49 de la LCE], el Consejo Vasco de Finanzas Públicas (art. 28 de la LTH), el Órgano de Relación; (art. 20 de la LCE) la Comisión Coordinadora (art. 40 de la LCE).

De todos los órganos de cooperación, participación o coordinación que se crean por parte del Gobierno Vasco, sólo se analizarán aquellos en los que de una forma u otra se encuentren representadas las Diputaciones Forales, ya que son las interrelaciones entre estos dos niveles los que ha suscitado más controversia.

Las Comisiones objeto de este análisis son 46 órganos que adquieren las siguientes denominaciones: comisiones, consejos, mesas, juntas, etc. Se ha considerado la denominación común de comisión para estos órganos por dos razones: en primer lugar, porque es la denominación que recoge la CORAME ${ }^{6}$, que ha sido el único estudio donde se ha encontrado una refe- rencia directa a los mismos. $Y$, en segundo lugar, porque es una de las denominaciones más comunes (21 comisiones, 16 consejos y 9 de otro tipo, como pueden ser mesas y juntas).

La investigación que justifica el presente artículo incluye una aportación basada en el análisis de documentos y una segunda parte en la que mediante el estudio del caso se pretende informar sobre el funcionamiento de los mismos.

\section{Caracterización de las comisiones}

\section{II.1. Distribución por departamentos de las comisiones}

A grandes rasgos podemos señalar que todos los departamentos del Gobierno Vasco crean comisiones ${ }^{7}$, y la mayoría de ellos cuenta con más de una. Los departamentos que cuentan con una única comisión son tres: Educación, Universidad e Investigación; Hacienda y Finanzas y, finalmente, el de Comercio, Consumo y Turismo.

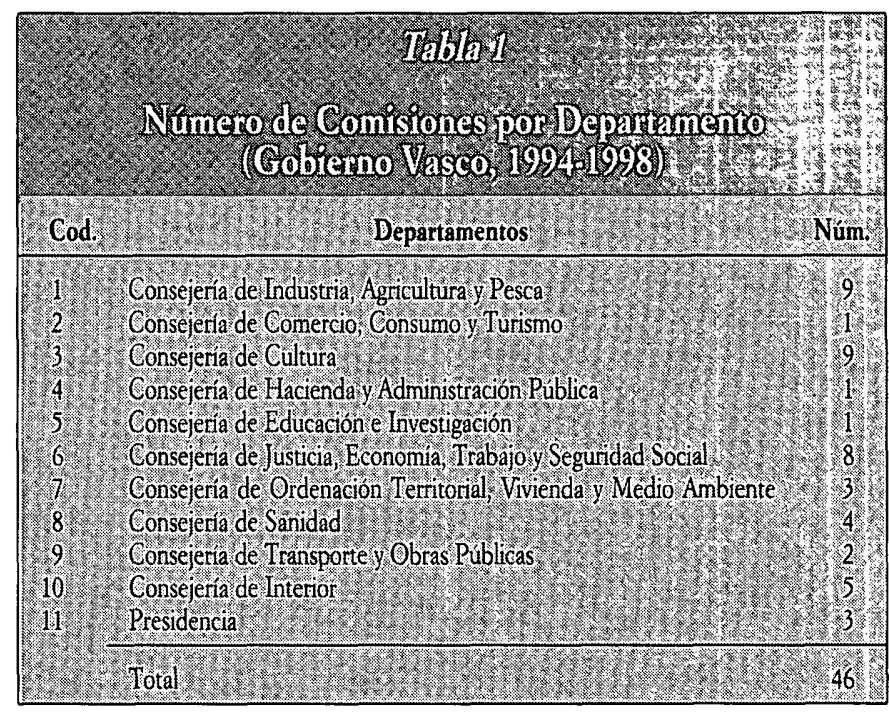

Fuente: Elaboración propia a partir de los decretos de estructura.

Los departamentos que cuentan con más comisiones son el Departamento de Industria, Agricultura y Pesca, y el de Cultura (9); seguidos de los Departamentos de Justicia, Economía, Trabajo y Seguridad Social (8). La razón de las variaciones en el número de comisiones dependiendo del Departamento no la encontramos en una política más abierta a la cooperación o participación de algunas Consejerías, sino más bien en las materias para las que se crean estas comisiones. Generalmente, 
las comisiones se crean para actuar en materias compartidas en las que las Instituciones Comunes (Gobierno y Parlamento) tienen competencia de emanar normas generales y los Territorios Históricos de desarrollar y ejecutar o simplemente de ejecución. También se da el caso de aquellas materias en las que aun siendo exclusivas de alguna de las instituciones, está muy relacionada con alguna otra exclusiva de la otra.

\section{II.2. Legislatura en la que se crean las comisiones}

La mayoría de las comisiones que se recogen en la quinta Legislatura de 1995 a 1998 son creadas durante la tercera Legislatura (16), y las demás son en su mayoría de la quinta Legislatura (15). Eso no significa que en las demás legislaturas no se hayan creado órganos de este tipo, o que no existieran en la primera Legislatura, dado que ha habido una evolución y existen comisiones que surgieron en los primeros años de la autonomía y que no han durado hasta esta quinta Legislatura que es la que estamos analizando.

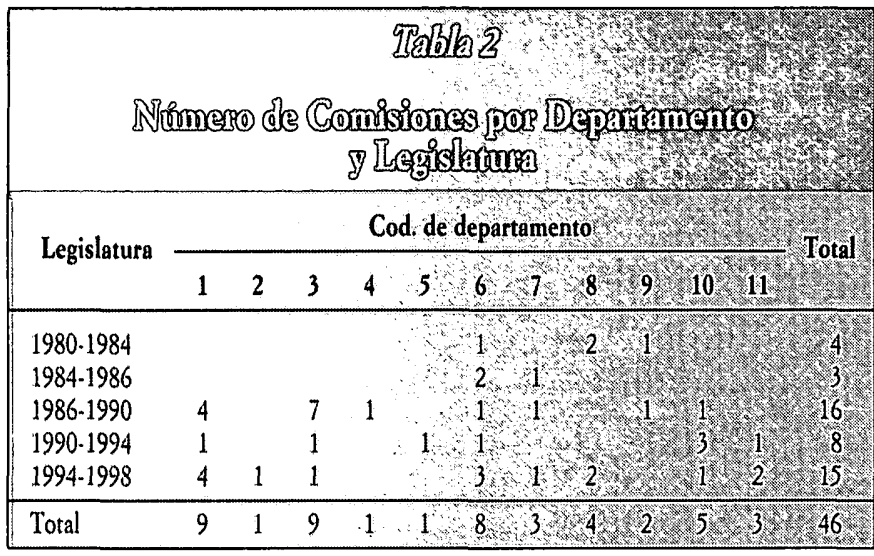

Fuente: Elaboración propia mediante los decretos de estructura.

El hecho de que la mayoría de las comisiones existentes en la quinta Legislatura fueron creadas en la tercera, no significa que sea en la tercera Legislatura cuando más comisiones se han creado, dado que en este estudio no se recogen aquellas comisiones que han sido sustituidas o disueltas y que, por tanto, no se adscriben a los Departamentos del Gobierno Vasco de la quinta Legislatura. Sin embargo, la tercera Legislatura se caracteriza por ser la primera después de la escisión en el Partido Nacionalista Vasco (PNV, en adelante). Uno de los factores que influyó en la división interna del PNV fue la LTH y, sobre todo, la definición del sistema institucional vasco y del reparto de competencias entre los poderes de la CAPV. La preocupación por el reparto de poder y las posibles disfunciones y malestares que la LTH había creado fue uno de los temas tratados en un informe $^{8}$ que se englobaba dentro del Acuerdo de Coalición entre PNV y PSE-PSOE cuando formaron gobierno.
Este informe es el resultado de una puesta en común de los problemas que no sólo el desajuste entre normativas creaba, sino también las disfunciones que las Diputaciones Forales y el propio Gobierno Vasco encontraban en la aplicación de la LTH. El informe final admite la existencia de problemas de aplicación, y termina señalando que tales problemas son solucionables a través de diferentes mecanismos entre los cuales se encuentran las comisiones, que enumera y cuyas futuras creaciones anuncia. Parece, por tanto, que la creación de tal número de comisiones en la tercera legislatura y su existencia puede ser justificada por la necesidad de dar una visión de coordinación dentro del sistema institucional vasco en un momento en el que éste estaba siendo puesto en duda.

\section{II.3. Composición de las comisiones}

Las comisiones, como órganos colegiados, se rigen por lo establecido en la Ley 30/1992 y, por tanto, se prevé la existencia de un/a presidente/a y secretario/a dentro de las mismas. Las comisiones están compuestas por: Presidente/a, Vicepresidente/a (no en todos los casos), secretario/a y vocales. El/La Presidente/a, Vicepresidente/a, si lo hay y el/la secretario/a son siempre representantes del Gobierno Vasco. Los/as secretarios/as suelen ser personal técnico del Gobierno Vasco y suelen tener voz pero no voto (en 38 de las 41 comisiones de la que se tiene información a este respecto).

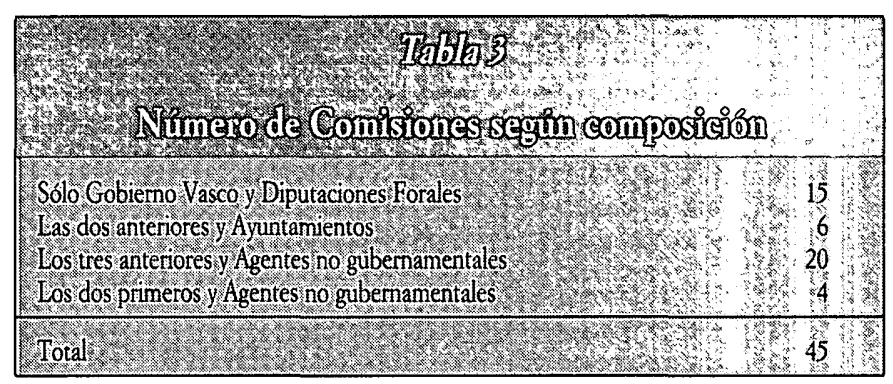

Fuente: Elaboración a partir de los Reglamentos internos y decretos de creación de las comisiones.

En cualquier caso, no puede considerarse al Gobierno Vasco como un actor homogéneo; por un lado, los intereses departamentales son diferentes y en muchos casos participan otros departamentos además de aquel al que el órgano está adscrito ${ }^{9}$, pero además el gobierno de la quinta Legislatura es un gobierno de coalición, con lo que a los intereses de cada materia se le suma los intereses partidistas. Por tanto, si analizáramos una decisión adoptada por uno de estos órganos, no deberíamos dar por supuesto que la voluntad o la posición del Gobierno Vasco fuera una, sino que deberíamos considerar los intereses departamentales y también el posicionamiento de los partidos políticos. 


\section{II.4. Finalidad y funciones de las comisiones}

Las comisiones son creadas para asesoramiento y consulta, se trata de órganos de información más que de participación y decisión, tal y como podremos comprobar tanto en el análisis de la finalidad como en el de las funciones. A continuación se enumeran las funciones de las comisiones:

a) Informar leyes, decretos, programas, proyectos o planes sobre una materia: casi todas las comisiones tienen la función de informar normas, planes o proyectos. Estos informes en su mayoría no suelen ser ni preceptivos ni vinculantes.

b) Proponer actuaciones a las instituciones o agentes sociales competentes en la materia: las comisiones suelen ser emplazadas a proponer actividades o programas dentro de la materia, pero también a proponer normas (su creación, revisión o modificación), criterios para elaboración de planes o valoración de la materia o medidas para el cumplimiento de las normas, programas o planes ${ }^{10}$.

c) Emitir informes sobre la materia: los informes que emiten las comisiones no siempre son requeridas para una norma o plan, sino que además tienen asignada la función de realizar estudios sobre la materia y emitir informes al Gobierno Vasco. Estos estudios pueden ser solicitados por el Gobierno Vasco, o por el departamento al que se adscribe la comisión, pero también pueden ser estudios que los componentes de la comisión consideren oportunos ${ }^{11}$.

d) Asesoramiento a las instituciones o a los agentes sociales sobre la materia: las comisiones, además de la labor de recopilación de información y emisión de informes, realizan una labor de asesoramiento sobre la materia, de forma habitual dirigida al Gobierno Vasco, pero en general para las instituciones y agentes sociales implicados.

e) Seguimiento y evaluación sobre la gestión de las instituciones en la materia.

f) Promoción de la Coordinación: un primer acercamiento a las comisiones nos hace pensar que son órganos de coordinación èntre el Gobierno Vasco y otras Instituciones pero esta función solamente se refleja en 11 de las 46 comisiones analizadas. Sin embargo, esta ausencia puede tener la siguiente explicación: la coordinación suele considerarse, en general, tal y como hemos visto, como una finalidad de las comisiones en sí misma, y no como una función particular del órgano ${ }^{12}$.

g) Otras: dentro de este apartado se introducen tanto las funciones como la elaboración del informe anual sobre las actividades de la comisión que se asigna a pocas comisiones $y$, por otro, aquellas funciones para las que se deja puerta abierta al Gobierno. Así, en 21 de las 46 comisiones estudiadas, se recoge como última función todas aquellas que el Gobierno Vasco les asigne o se les asigne reglamentariamente.

\section{II.5. Convocatorias de las comisiones}

Las comisiones establecen un orden de convocatoria generalmente anual, y la posibilidad de convocar extraordinariamente la comisión siempre que el/la presidente/a lo considere oportuno o cuando un número preestablecido de vocales o miembros de la comisión lo consideren necesario.

Las convocatorias ordinarias: no todas las comisiones establecen un mínimo de convocatorias ordinarias. Las que recogen en sus reglamentos un número de convocatorias ordinarias anuales mínimas, éste no es el mismo para todas. El mínimo establecido oscila desde una vez al año (cada doce meses) hasta cuatro veces al año (trimestralmente), ninguna de las comisiones prevé un número mínimo de convocatorias anuales superior a cuatro.

Las convocatorias extraordinarias: suelen ser establecidas por el presidente/a, que es la única persona responsable de convocar la comisión. Esto no significa que sólo se reúnan con carácter extraordinario cuando el/la presidente/a lo considere oportuno, ya que algunas comisiones recogen la posibilidad de que con la solicitud de una cantidad de miembros, el/la presidente/a deba convocar la comisión.

\section{II.6. La toma de decisión en las comisiones}

La toma de decisiones se lleva a cabo de tres maneras dependiendo de la comisión de la que se trate: mayoría simple, mayoría absoluta y unanimidad. De todos los casos analizados sólo en uno es necesaria la unanimidad, es el caso de la Comisión Ambiental del País Vasco. La mayoría absoluta se exige en 7 de los 31 casos sobre los que se dispone de información, a este respecto y en los demás la mayoría simple.

Por tanto, en general se sigue lo establecido por la Ley 30/1992, de Régimen Jurídico de las Administraciones Públicas y del Procedimiento Administrativo Común (Ley 30/1992, en adelante) para órganos colegiados ${ }^{13}$.

\section{II.7. Práctica de las comisiones}

Tal y como veíamos al analizar las convocatorias, ninguno de estos órganos se reúne más de 4 veces al año, salvo convocatorias extraordinarias, por tanto, parece que el trabajo que puedan realizar éstos como participación o coordinación es limitada. Más aún, si analizamos las veces en las que se han podido convocar a estos órganos en esta legislatura, podemos señalar que es escasísima la funcionalidad de estos órganos en la práctica. Así, entre las razones de la escasa funcionalidad de estas comisiones se encuentran las siguientes: 
- Por creación reciente: los órganos tienen un proceso de creación y formación que suele dilatarse en el tiempo. El proceso comienza con su creación por ley o decreto, y es éste el período que recogemos como momento de creación, sin embargo, no es una creación en la práctica. Una vez que el Gobierno Vasco o alguno de sus departamentos establece la configuración de un órgano, señalando la composición de los mismos, debe informar a todos los agentes que tienen representación para que elijan los vocales que les corresponden. Una vez establecidos los vocales hay un período para su primera reunión donde se establece la creación real, y donde suelen recogerse los temas relativos a su organización. Por tanto, los órganos que se crean en la última Legislatura, es poco probable que se hayan reunido más de dos veces durante la misma y, por tanto, podemos decir que prácticamente funcionarán en la sexta Legislatura. De las 46 comisiones analizadas, 15 de ellas se crean en la quinta Legislatura, es decir, entre 1995 y 1998, pero además 9 de ellas se han creado entre 1997 y 1998 lo que supone un nivel de funcionalidad baja en la Legislatura que analizamos.

- Por inactividad: se refiere a las creadas con anterioridad, y que aun apareciendo en los decretos de estructura no se han reunido. Así, las comisiones, a pesar de crearse, de estar establecidas en los decretos de estructura, no siempre se encuentran en activo, hay comisiones que se han creado en la segunda, tercera o cuarta Legislatura, que no se reúnen con regularidad. Sin haber realizado un estudio exhaustivo de todas las comisiones existentes en cuanto a si se convocan o no, lo cierto es que reconocemos cinco casos sobre los no ha habido actividad: el Consejo General de Cultura, que se creó en 1987, no se ha convocado en ocho años. Lo mismo ocurre con las Juntas Asesoras de Danza y Folklore, Cinematografía, Teatro y Música.

- Por desaparición: en este grupo se hallan aquellas comisiones que han desaparecido o se han disuelto, por ser sustituidas por otras o simplemente porque no eran necesarias. Las comisiones no son estables ni permanentes, y pueden crearse en una legislatura y desaparecer en la siguiente, porque responden, generalmente, a las necesidades de organización o de programa político del partido gobernante en la Legislatura. Eso significa que si es limitada la labor de las comisiones que se crean al comienzo de una Legislatura, también lo es la de aquellas que desaparecen a mitad de Legislatura. Por ejemplo, en materia de agricultura existían comisiones que han sido sustituidas por otros órganos, así, la Comisión de Agricultura de Montaña ha perdido su funcionalidad porque el trabajo que ésta realizaba lo lleva a cabo la Sociedad Pública MENDIKOI, S. A. Asimismo, en materia de Sanidad, la Junta Vasca de la Salud, existente desde 1983, si nos remitimos a la fecha de su creación, se disuelve con la nueva Ley 8/1997, de 26 de junio, de Ordenación Sanitaria del País Vasco, que crea el Consejo de Sanidad del País Vasco.
Podemos concluir este apartado señalando que 21 de las 46 comisiones analizadas no han realizado una práctica completa, en cuanto a que no se han podido reunir, por las razones ya citadas.

\section{Estudio de casos}

El Consejo de Conservación de la Naturaleza, la Comisión Ambiental del País Vasco, el Consejo de Función Pública Vasca y el Consejo Asesor del Euskera.

Para el estudio de los casos, hemos elegido cuatro de las 46 comisiones recogidas en las que participan las Diputaciones Forales. Los criterios de selección han sido los siguientes: 1 . Ser una comisión que haya tenido un mínimo de convocatorias; 2. Ser una comisión que tenga una finalidad genérica (que no se limite a una actividad concreta como puede ser la de aprobar una plan); 3. Mostrar colaboración y accesibilidad, y 4. Pertenecer a diferentes departamentos.

\section{III.1. Consejo de Conservación de la Naturaleza: Naturzaintza}

El Consejo de Conservación de la Naturaleza (CCN, en adelante) fue creado por la Ley 16/1994, de Conservación de la Naturaleza, como órgano consultivo y de cooperación en la materia. En cuanto a su composición no es un órgano simplemente interinstitucional, ya que en él participan además de representantes del Gobierno Vasco, las Diputaciones Forales y los Ayuntamientos, los agentes sociales implicados con la materia. En las diferentes convocatorias que se han llevado a cabo los vocales han asistido con asiduidad. Las convocatorias de esta comisión ha superado lo reglamentariamente establecido, lo que no es usual en estos casos (en tres años se han reunido nueve veces).

El reglamento interno de este órgano establece que los acuerdos se adoptarán por la mitad más uno de los miembros del Consejo presentes. En la práctica el consenso de los miembros ante los acuerdos, informes, planes, etc., ha sido mayor, ya que en la mayoría de los casos el resultado de las votaciones es de unanimidad, además debemos sumar a éstos aquellos en los que, sin haber unanimidad, no hay voto en contra, sino abstención.

De las funciones estandarizadas para estos órganos recogidas en el apartado dedicado a las funciones y fines, el CCN no recoge todas como propias en su reglamento interno; así, no tiene funciones de asesoramiento a las instituciones o agentes sociales. Sin embargo, las funciones puestas en práctica difieren 
un poco de lo recogido en el anterior apartado, si tenemos en cuenta las actas de las reuniones. De acuerdo con la clasificación funcional que se recoge para las comisiones en general, podemos decir que, en la quinta Legislatura, el CCN ha ejercido las siguientes funciones:

1. Informar leyes, decretos, programas o proyectos o planes sobre una materia: el CCN ha informado favorablemente once decretos, programas, proyectos y planes como: el Plan de Uso Público de Parques Naturales, el Plan de ordenación de Recursos Naturales, etc.

2. Seguimiento y evaluación de las actuaciones en la materia: esta función que tiene asignada el $\mathrm{CCN}$ no ha sido apenas ejercida y se limita a un debate sobre la situación del lobo y la situación de los parques eólicos en la CAPV.

3. Proponer actuaciones a las instituciones o agentes sociales competentes en la materia: se traslada al Departamento de Ordenación del Territorio, Vivienda y Medio Ambiente el interés de $\mathrm{CCN}$ por conocer el estado de la tramitación del proyecto de Ley de Medio Ambiente de la Comunidad Autónoma del País Vasco. Propuestas de lugares de la CAPV susceptibles de ser considerados como zonas especiales de conservación para su inclusión en la red ecológica europea «Natura 2000».

Podemos señalar, como conclusión, que si bien se cumple la norma en cuanto a convocatoria y composición, lo cierto es que en cuanto a las funciones puestas en práctica la utilización de este consejo ha sido para el asesoramiento y consulta del Gobierno Vasco. El CCN, por su práctica, es un órgano en el que el Gobierno Vasco reúne a las diferentes instituciones públicas y agentes sociales interesados en la materia para informarles sobre las actuaciones del Gobierno Vasco, dándoles la posibilidad de emitir su opinión sin que esto obligue al Gobierno Vasco a nada. Respecto al posicionamiento de las Diputaciones Forales, hay que señalar su acuerdo en la mayoría de los Planes, lo que puede hacer sospechar que exista un previo acuerdo entre el Gobierno Vasco y las Diputaciones Forales.

\section{III.2. La Comisión Ambiental del País Vasco}

La Comisión Ambiental del País Vasco (CADPV) es un órgano creado por decreto en 1989 , como instrumento de relación y participación de las distintas administraciones que en el área del Medio Ambiente actúan en la Comunidad Autónoma del País Vasco. Se trata de una comisión interinstitucional donde no tienen cabida los/as agentes sociales como miembros del mismo.

En 1997 se aprueba la Ley de Medio Ambiente, donde se recoge la constitución del CADPV, pero se prevé en la misma algunos cambios para esta comisión, sobre todo en lo que a su composición se refiere. Sin embargo, esta nueva Comisión no se ha reunido aún porque la designación de sus represen- tantes se encuentra actualmente en trámites. Por tanto, la que estudiaremos a continuación es la existente hasta 1997.

Desde su creación en 1989, la CADPV se ha reunido cuatro veces en pleno, con lo que en general la actividad de esta comisión ha sido reducida. Si analizamos, tal y como estaba previsto, la quinta Legislatura, sólo se reduciría a una reunión (7 de noviembre de 1996), por lo que, a pesar de hacer hincapié en esta Legislatura, vamos a analizar las cuatro sesiones plenarias para poder valorarla en cuanto a sus funciones.

La presidencia de la CADPV la ostenta la Viceconsejera de Medio Ambiente, ejerciendo como tal en todas las convocatorias de la CADPV realizadas hasta el momento. El Gobierno Vasco cuenta además con otros dos representantes, que son: el Director de Calidad Ambiental y la Directora de Recursos Ambientales, participando, ambos, de forma activa en la CADPV. Las Diputaciones Forales cuentan con 3 representantes; uno por cada Territorio Histórico. La representación de las Diputaciones Forales es ostentada por los Diputados Forales competentes en Medio Ambiente; sin embargo, éstos suelen ser sustituidos por Directores del área medioambiental. A los ayuntamientos les corresponde un solo representante, que suele ser elegido por la Asociación Vasca de Municipios (EU. DEL).

Según su reglamento interno, el pleno será convocado por su Presidente, al menos una vez cada dos meses. Sin embargo, la realidad práctica es otra y, como hemos podido comprobar, la CADPV ha tenido sólo una reunión anual. Por su parte, no se ha podido comprobar si las decisiones han sido adoptadas por unanimidad, ya que en las actas no encontramos reflejo de votación. El CADPV tiene asignadas las siguientes funciones:

1. Informar leyes, decretos, programas, proyectos o planes sobre una materia: ésta es la función que más tiempo les ha llevado, si tenemos en cuenta las cuatro reuniones. A pesar de que la capacidad de actuación de la CADPV es nula, y que tiene una posición pasiva, dado que no es una actuación o decisión que adopta este órgano, sino que sólo es informado. Todos estos planes y proyectos son del Gobierno Vasco, a pesar de que, en general, las diferentes instituciones dan su opinión sobre lo mismo, lo cierto es que en una sola ocasión el representante del Gobierno Vasco ha considerado la posibilidad de realizar reformas en el proyecto ${ }^{14}$.

2. Proponer actuaciones a las instituciones o agentes sociales competentes en la materia: los diferentes miembros a nivel individual proponen sobre todo al Gobierno Vasco algunas actuaciones en materia concreta, como educación ambiental, pero no se toma una decisión común. Las propuestas suelen realizarse de forma unidireccional, desde las Diputaciones Forales o desde EUDEL al Gobierno Vasco.

3. Promoción de la coordinación: la promoción de la coordinación se ha reducido en la práctica a tres actuaciones con- 
cretas, que por el formalismo de las mismas son muy criticables en cuanto a su funcionalidad:

a) La puesta en común de los anteproyectos de presupuestos: esta práctica se ha realizado en dos ocasiones, con los presupuestos de 1993 y con los de 1997, según se tiene constancia en las actas. Además de ser criticable lo esporádico de estas actuaciones, también puede ponerse en duda la funcionalidad de las mismas. La presentación de los anteproyectos presupuestarios se lleva a cabo de dos formas, según se trate del Gobierno Vasco o de las Diputaciones Forales. El Gobierno Vasco explica o expone el anteproyecto mientras que las Diputaciones Forales se limitan a repartir una copia de los mismos a cada uno de los miembros. La información que se da en los presupuestos sobre la actuación de las diferentes instituciones es muy limitada, dado que, aun tratándose de la parte del presupuesto por programas, éstos suelen ser poco definitorios de la realidad de las actuaciones de las instituciones.

b) Exposición de la política medioambiental de las diferentes Administraciones Públicas de la CAPV: éste fue uno de los puntos del orden del día de la última reunión celebrada por la CADPV, cuyo desarrollo se redujo a lo siguiente: la Viceconsejera de Medio Ambiente explicó la actuación del Gobierno Vasco en Medio Ambiente, pero sin entrar a profundizar en ningún elemento. Por su parte, los/as diferentes representantes de las Diputaciones Forales aportaron el decreto de estructura del departamento competente en esa materia, y las funciones normativamente asignadas por estos decretos a los diferentes órganos de las Diputaciones que tienen responsabilidad en materia ambiental. EUDEL (la Asociación de Municipios del País Vasco), por su parte, se limitó a señalar la necesidad de establecer nítidamente las funciones y competencias de los diferentes niveles de gobierno de la CAPV, y explica algunos de los problemas con los que se encuentran los ayuntamientos en la gestión de medio ambiente.

c) Actuaciones concretas: la existencia de problemas en la gestión de residuos sólidos en la cuenca del Nervión es debatida en la CADPV. Sin embargo, al tratarse de un problema local que no afecta a todos los miembros del mismo, sino a unos actores específicos que no se encuentran en su totalidad representados en la comisión, se acuerda la creación de un nuevo órgano interinstitucional coordinado por la Dirección de Calidad Ambiental, que tratará sobre el tema. El problema de gestión de residuos sólidos no afecta al Gobierno Vasco como tal (por eso realizará labores de arbitraje), sino a las Diputaciones Forales de Bizkaia y Araba y al Ayuntamiento de Gordexola.

La CADPV es limitada como órgano de participación, dado que no se interviene en la toma de decisión, ni en la elaboración de los planes o los decretos, simplemente se limitan a dar su opinión sobre el texto global. Como órgano de coordinación, no es suficiente, dado que la información de los presupuestos y de los decretos de estructura no son más que la galería de las actuaciones de las administraciones. Sin embargo, no hay que restarle importancia, dado que se trata de un paso adelante en la coordinación.

\section{III.3. El Consejo Vasco de Función Pública}

El Consejo Vasco de Función Pública (CVFP) se crea con la Ley de Función Pública Vasca en $1989^{15}$, y al igual que ésta se modifica en $1997^{16}$. Desde 1995, cuando comienza la quinta Legislatura, hasta el final de la misma en 1998, el CVFP se ha reunido en cinco ocasiones, una vez al año. Esta comisión agrupa a las diferentes instituciones públicas de la CAPV, como son: el Gobierno Vasco, las Diputaciones Forales y los ayuntamientos. No es, sin embargo, solamente un órgano interinstitucional, dado que faculta la participación como miembros de este órgano a agentes sociales; en este caso, concretamente a los sindicatos.

El Gobierno Vasco está representado, además de por el presidente (cargo que corresponde al Consejero de Hacienda y Administración Pública en la quinta Legislatura), por otros tres miembros, a los que no se atribuye ninguna exigencia de rango o materia. Han ocupado esta representación: el Director de Gestión de Personal de Osakidetza, el Director de Gestión de Personal de Educación, Universidades e Investigación y el Director del Instituto Vasco de Administración Pública. Las Diputaciones Forales tienen asignados tres representantes, sin exigir de los mismos un rango. La representación ha sido llevada a cabo por los Diputados Forales de Presidencia o cargo equivalente. La presencia de los mismos ha sido intermitente en los tres casos. Los ayuntamientos, al igual que ocurriera con la Comisión Ambiental del País Vasco, están representados por EUDEL, pero en este caso cuentan con tres representantes, que se repiten en las diferentes sesiones. Generalmente, han asistido dos, salvo en una ocasión en la que se encontraron presentes los tres.

Los sindicatos representados en el CVFP son ELA, LAB, CCOO y UGT. ELA cuenta con cuatro representantes, LAB con tres, CCOO y UGT con uno cada uno. En total suman siete representantes sindicales, a pesar de que la norma recoge que deberían de ser seis. En general, todos se han presentado a las reuniones, salvo en la del día 9 de abril de 1997, y lo hacen como señal de protesta.

Tal y como ocurre en la comisión anteriormente estudiada, la convocatoria no se cumple y se reúnen en menos ocasiones que las establecidas normativamente. La norma establece que el CVFP se reunirá cuatro veces al año de forma trimestral. Según la norma que regula el funcionamiento de este órgano, las decisiones se adoptan por mayoría simple. A pesar de ello, en general el consenso ha sido mayor. En el caso del CVFP, 
los votos en contra han provenido de los sindicatos principalmente y sólo en tres de las ocho votaciones realizadas ha habido algún voto en contra desde alguna de las Diputaciones Forales ${ }^{17}$. La importancia de que la votación sea a favor de un informe favorable de la norma es relativa, dado que, si bien es preceptiva, no es vinculante la decisión.

Las funciones reglamentariamente asignadas se resumen en las siguientes tres funciones estandarizadas: 1. Informar leyes, decretos, programas, proyectos o planes sobre una materia; 2. Proponer actuaciones, y 3. Emitir informes sobre la materia. Sin embargo, la actividad de este CVFP está poco relacionada con lo establecido por la norma reguladora en la quinta Legislatura, ya que en la práctica se ha reducido su función a la información de normas y todas ellas procedentes del Gobierno Vasco.

Recapitulando, el CVFP se ha dedicado a la emisión de informes sobre los proyectos del Gobierno Vasco. A pesar de que los informes sólo eran preceptivos, en todos los casos han sido favorables. Existe cierto consenso entre las posiciones del Gobierno Vasco y las Diputaciones Forales. Esta forma de actuación es más informativa que coordinadora, participativa, asesora o consultiva.

\section{IIII.4. Consejo Asesor de Euskera}

El Consejo Asesor de Euskera (CAEU, en adelante) fue creado en 1982 por la Ley 10/1982, de 24 de noviembre, Básica de Normalización del Uso del Euskera. La última modificación de este órgano se realizó en 1996 con el D. 156/1996, de 18 de junio, de Transformación del Consejo Asesor de Euskera. Este órgano se configura como órgano de encuentro para el estudio, canalización y coordinación de los esfuerzos y actividades de las diversas instituciones, tanto públicas como privadas, implicadas en la normalización lingüística.

En la quinta Legislatura se han reunido en pleno en cinco ocasiones, y en comisiones permanentes o especiales quince veces. En lo referente a la composición, el Gobierno Vasco cuenta con nueve vocales, que representan diferentes departamentos y entidades. Son tres los departamentos representados: la Consejería de Cultura, la Consejería de Hacienda y Administración Pública y la Consejería de Educación, Universidades e Investigación. Además de los departamentos, participan también en este foro las siguientes entidades: HABE (Instituto de Alfabetización y Reeuskaldunización de Adultos), EITB (Eukal Irrati Telebista), Euskaltzaindia. A las Diputaciones Forales se les asigna un representante por Territorio Histórico. Araba y Gipuzkoa han participado en el órgano representándose por un/a director/a, mientras que ha sido el Diputado Foral de Cultura el que ha participado por parte de la Diputación Foral de Bizkaia. La Administración municipal está representada de dos formas: por un lado, y de forma general, por EUDEL y, por otra, de forma particular, mediante el representante de la Mancomunidad de Municipios Euskaldunes (UEMA, en adelante). Tanto a EUDEL como a UEMA les corresponde la elección de un/a vocal para el CAEU.

Las convocatorias establecidas como mínimas en la normativa que regula el funcionamiento del CAEU han sido cumplidas en el caso de este órgano. El órgano, si bien se creó en los años ochenta, no ha tenido un funcionamiento regular desde el período de creación hasta el momento. La puesta en funcionamiento de nuevo de este órgano se produce con el Decreto de 1996 ya mencionado, y, por tanto, es a partir de esta fecha cuando se producen las reuniones. Las funciones del CAEU son las siguientes:

1. Informar leyes, decretos, programas o proyectos o planes sobre una materia: el CAEU debe informar el borrador del Plan General de Promoción del Uso de Euskera que elabore la Viceconsejería de Política Linguística con carácter previo a su presentación al Consejo de Gobierno Vasco. Este informe no tiene carácter preceptivo ni vinculante, ya que no se recoge como tal en la normativa.

2. Proponer actuaciones a las instituciones o agentes sociales competentes en la materia: esta comisión tiene asignada como función la de proponer al Gobierno y a las Instituciones correspondientes la adopción de medidas adecuadas para la mejor aplicación de las disposiciones referentes al uso del Euskera.

3. Emitir informes sobre la materia: además de informar el Plan General del Uso del Euskera, el CAEU deberá estudiar y analizar, a instancias del Gobierno Vasco, cuantas cuestiones se refieran a la normalización lingǘstica en la CAPV.

4. Seguimiento y evaluación de las actuaciones en la materia: el CAEU, una vez aprobado el Plan General de Uso del Euskera, deberá realizar una memoria anual, evaluando el desarrollo de dicho Plan. Además, deberá presentar una memoria anual de evaluación de la actividad de su comisión permanente.

5. Promoción de la coordinación: la coordinación se recogía como una de las finalidades de este órgano, y así se refleja también en la lista de funciones asignadas al mismo. El CAEU deberá proponer medidas que aseguren la actuación coordinadora de todos los poderes públicos con competencia en materia lingüística.

Las funciones o actividades llevadas a cabo por este órgano en el período 1995-1998 difieren un poco de lo recogido con anterioridad. Su labor se ha centrado en su organización interna, y la colaboración en la elaboración del Plan General de Promoción del Uso del Euskera. La colaboración es tan amplia que se introducen dentro de ese marco todas las demás funciones: evalúan la actuación de los entes públicos y de la propia comisión, se realizan estudios contando con la presencia de experiencias exteriores, se proponen actuaciones y, finalmente, se informa favorablemente el Plan. 
El CAEU es un órgano que funciona en pleno, pero también en comisiones permanentes o especiales. En la nueva etapa de este órgano, desde 1996, el funcionamiento ha sido el siguiente: por un lado, se ha creado una Comisión Permanente que se ha reunido de forma regular durante toda la etapa analizada, y, por otro lado, se han creado comisiones especiales que han variado. En un primer momento y como paso previo para la elaboración de Plan de Promoción del Uso del Euskera, se crean tres comisiones especiales: 1. Medios Audiovisuales y Culturales, 2. Prensa, Publicaciones y Ediciones, y 3. Continuidad del Euskera. Una vez aprobado el Plan al que hacíamos referencia, se entra en una segunda etapa donde se disuelven las anteriores comisiones especiales y se crean dos nuevas: de seguimiento y de desarrollo normativo.

El CAEU es la comisión de los casos de estudio realizados que funciona con más regularidad en cuanto a sus reuniones y también a la funcionalidad de las mismas. Sin embargo, se debe tener en cuenta que la finalidad que ha llevado a la actividad dinámica de esta comisión es la elaboración del Plan de Promoción del Uso del Euskera; por tanto, otro estudio debería de encargarse de analizar si, una vez elaborado y aprobado éste, el CAEU tendrá continuidad en la implementación del Plan.

Tal y como ocurre en las demás comisiones, el Gobierno Vasco, que es quien crea el órgano, dirige todas las actuaciones de la comisión a través de la Comisión Permanente y de la Dirección de Política Lingüística que filtra (es decir, no sólo traspasa la información de las comisiones permanentes al pleno, sino que selecciona aquellas propuestas que considera más adecuadas de todas las propuestas por las comisiones especiales y son, únicamente, éstas las que lleva al pleno) la información recogiendo en el Plan aquello que considera oportuno.

\section{Conclusión}

Las comisiones son instrumentos formales de coordinación, participación y asesoramiento entre los diferentes órganos institucionales y agentes sociales. El objeto de estudio, no obstante, se ha centrado en aquellas comisiones creadas por el Gobierno Vasco en el que las Diputaciones Forales tienen participación. Las comisiones, que tienen como finalidad la participación, el asesoramiento y la coordinación, son órganos necesarios en un sistema institucional tan descentralizado como el de la CAPV, $y$, por tanto, debemos señalar la conveniencia de la existencia de éstas.

La variedad de comisiones con las que cuenta la quinta Legislatura deja de manifiesto la voluntad del Gobierno Vasco de dar participación a las demás instituciones en la elaboración de políticas comunes, pero también en recabar la posición de personas expertas y agentes sociales que trabajan en la materia. Sin embargo, la dinámica de estos órganos nos lleva a considerar la necesidad de variaciones en su funcionamiento si se pretende lograr la participación, la coordinación y la búsqueda de asesoramiento por parte de los mismos. Destacamos que:

1. Las comisiones se caracterizan por una fuerte dependencia del Gobierno Vasco, sin que se cree en ella una vida propia independiente de la institución: son creadas por el Gobierno Vasco; éste define la parte más importante de la organización interna del órgano y decide convocarla o no, sin que ésta cumpla ni siquiera lo establecido normativamente como mínimo. Finalmente, suele ser éste el que establece el orden del día.

2. Las decisiones adoptadas en el seno de las comisiones no exigen en general más que la mayoría simple; sin embargo, en la práctica el consenso que se logra es mayor. Destacaría el posicionamiento de las Diputaciones en las que se ha detectado un acuerdo dominante con casi todas las propuestas realizadas desde el Gobierno Vasco. Esto puede deberse a tres cosas: la coincidencia, en realidad, entre las posiciones del Gobierno Vasco y las Diputaciones, la existencia de acuerdo previo (funcionamiento de instrumentos informales de relación y coordinación) y la consideración de que la decisión no tiene efectos sobre sus competencias o recursos. Las razones variarían según la decisión, pero consideramos que las dos últimas reflejan mejor la realidad.

3. Tal y como señalamos en el punto anterior, las decisiones han sido favorables en relación a las propuestas del Gobierno Vasco, sin embargo, de no serlo no tendría gran transcendencia, dado que estas decisiones adoptadas por las comisiones, en general, no tienen carácter ni perceptivo ni vinculante.

4. Las convocatorias de las comisiones adquieren importancia, ya que, si no se producen, el órgano deja de cumplir con sus funciones de participación y coordinación, no así la de asesoramiento, dado que el Gobierno Vasco convocará al órgano siempre que necesite su punto de vista. La realidad muestra que los órganos no tienen un funcionamiento regular en general y que no cumplen con las convocatorias ordinarias normativamente establecidas.

5. Las funciones atribuidas a las comisiones normativamente pueden considerarse generosas en cuanto a que se les atribuye, además de informar normas, planes, proyectos, etc., elaborados por las distintas administraciones, la posibilidad de proponer actuaciones, realizar estudios y evaluaciones $\mathrm{y}$, por último, promover la coordinación entre instituciones en la materia. Sin embargo, en los casos estudiados, la función dominante ha sido la de informar los proyectos, planes y normas presentados por el Gobierno Vasco. No obstante, hay que destacar la voluntad de coordinación, mediante iniciativas como la comparación de presupuestos o creación de órganos dentro de las comisiones de coordinación. 
Las comisiones cumplen un papel de asesoramiento o consulta al servicio del Gobierno Vasco y de información a las demás instituciones y agentes sociales sobre las actividades del Gobierno Vasco; sin embargo, se considera escaso el valor de éstas como órganos de coordinación. A pesar de que la voluntad de las normas que las regulan es clara en este sentido, la práctica muestra que no se ha desarrollado la posible potencialidad de las comisiones.

\section{Anexo I. Las Comisiones, Consejos y Juntas de relación entre TTHH y Gobierno Vasco, que se crean para la Legislatura 1994-1998}

1. Consejería de Industria, Agricultura y Pesca:

- Consejo Asesor de la Naturaleza.

- Comisión de Política Agraria.

- Comisión de Política Pesquera.

- Comisión de Agricultura de Montaña.

- Mesa Consultiva Nacional Agraria.

- Mesa Consultiva Nacional de la Pesca.

- Comisión de Productos Zoosanitarios.

- Comisión de Asesoramiento Técnico Control Antidoping.

- Landaberri.

- Consejo Consultivo de Desarrollo Rural.

2. Consejería de Comercio, Consumo y Turismo:

- Comisión Consultiva de Comercio.

3. Consejería de Cultura:

- Consejo General de Cultura Vasca.

- Consejo Vasco del Deporte.

- Comité contra la Violencia en el Deporte.

- Consejo Asesor del Patrimonio Arquitectónico Monumental.

- Consejo Asesor de Euskera.

- Junta Asesora de la Danza y el Folklore.

- Junta Asesora de Cinematografía.

- Junta Asesora de Teatro.

- Junta Asesora de Música.
4. Consejería de Hacienda y Administración Pública:

- Consejo Vasco de Función Pública.

5. Consejería de Educación e Investigación:

- Consejo Vasco de Formación Profesional.

6. Consejería de Justicia, Economía, Trabajo y Seguridad Social:

- Comisión Vasca de Estadística.

- Consejo Vasco de Estadística.

- Consejo Vasco de Bienestar Social.

- Comisión Técnica de Adopción Internacional.

- Comisión Interinstitucional para la Inserción.

- Consejo Vasco del Voluntariado.

- Comisión de Acreditación de Servicios Residenciales de la Tercera Edad.

- Grupos Funcionales de Planificación Económica.

7. Consejería de Ordenación Territorial, Vivienda y Medio Ambiente:

- Comisión de Ordenación del Territorio del País Vasco.

- Comisión Ambiental del País Vasco.

- Consejo Vasco para la Promoción de la Accesibilidad.

- Consejo Asesor de Drogodependencias.

8. Consejería de Sanidad:

- Comisión para el Estudio y Racionalización de la Red de Transporte Sanitario de Urgencia.

- Junta Vasca de la Salud.

- Consejo de Sanidad del País Vasco.

9. Consejería de Transporte y Obras Públicas:

- Comisión Vasca de Coordinación de Transporte de Mercancías Peligrosas.

- Comisión del Plan General de Carreteras.

10. Consejería de Interior:

- Comisión Vasca para la Seguridad.

- Comisión de la Protección Civil del País Vasco.

- Comisión de Seguridad Vial.

- Comisión Vasca Asesora para Asuntos Taurinos.

- Consejo Vasco del Juego.

11. Presidencia: Vascas.

- Consejo Asesor de Relaciones con las Colectividades

- Comisión Gestora del Fondo para la Cooperación y Ayuda al Desarrollo del Tercer Mundo y Técnica de Apoyo y Asistencia.

- Consejo Asesor de Cooperación al Desarrollo del País Vasco. 
* Becaria Predoctoral del Gobierno Vasco adscrita al Departamento de Ciencias Políticas y de la Administración. Facultad de Ciencias Sociales y de la Comunicación. Ministerio del País Vasco.

${ }^{1}$ Ley 27/1983, de 25 de noviembre, de Relaciones entre las Instituciones Comunes de la Comunidad Autónoma y los Órganos Forales de sus Territorios Históricos, BOPV, núm. 182, de 10 de diciembre de 1983.

${ }^{2}$ Gobierno Vasco. Informe sobre el marco institucional interno elaborado de conformidad con lo previsto en el Epígrafe II, apartado $4 .^{\circ}$, del Programa de Gobierno, Vitoria-Gasteiz, abril de 1989.

${ }^{3}$ Ley Orgánica 3/1979, de 18 de diciembre, de Estatuto de Autonomía para el País Vasco, BOE, núm. 306, de 22 de diciembre de 1979.

+ Ley 27/1983, de 25 de noviembre, de Relaciones entre las Instituciones Comunes de la Comunidad Autónoma y los Órganos Forales de sus Territorios Históricos, BOPV, núm. 182, de 10 de diciembre de 1983.

${ }^{5}$ Ley $12 / 1981$, de 13 de mayo, por la que se aprueba el Concierto Económico de la Comunidad Autónoma del País Vasco, BOE, núm. 127, de 28 de mayo de 1981.

6 Gobierno Vasco, Informe de la Comisión para la Racionalización y Mejora de la Administración Pública, 21 de junio de 1994.

7 El nombre de las comisiones por departamento puede consultarse en el anexo núm. 1.

${ }^{8}$ Gobierno Vasco, Informe sobre el marco institucional interno elaborado de conformidad con lo previsto en el Epígrafe II, apartado $4 .^{\circ}$, del Programa de Gobierno, Vitoria-Gasteiz, abril de 1989.

9 (No propondrá las mismas medidas en el tema de la drogodependencia el Departamento de Sanidad que el de Interior.)

${ }^{10}$ Por ejemplo: el Consejo Vasco de Formación Profesional tienen como funciones: Proponer al Gobierno Vasco (...) la determinación de los programas y contenidos mínimos homogeneizadores (...); Proponer al Gobierno Vasco (...), los criterios generales para la determinación de los complementos específicos y de productividad en las distintas Administraciones Públicas Vascas; Proponer medidas dirigidas a la euskaldunización de personal al servicio de las Administraciones Vascas; (...) Proponer al Presidente el encargo de estudios, trabajos, informes, etc., a profesionales (...).

${ }^{11}$ Por ejemplo: la Comisión para el Estudio y Racionalización de la Red de Transporte Sanitario de Urgencia tiene como funciones: estudio y evaluación de recursos técnicos y humanos relacionados con el Transporte Sanitario de Urgencia, emisión $\mathrm{de}$ informes y dictámenes a petición de los respectivos departamentos del Gobierno y Diputaciones Forales, (...); Conocimiento y análisis de la gestión de los servicios de transporte sanitario de urgencia.

${ }^{12}$ Por ejemplo: la Comisión Ambiental tiene las siguientes funciones de coordinación: Promover la coordinación y realizar las labores de seguimiento pertinentes de todas aquellas actuaciones que en el área del medio ambiente sean desarrolladas por las Instituciones representadas en la Comisión.

${ }^{13}$ Art. 26.4 de Ley 30/1992. «Los acuerdos serán adoptados por mayoría de votos.»

${ }^{14}$ Concretamente se trata del Plan de Gestión de Residuos Inertes, presentado a la CAPV el 1 de julio de 1994. Las alegaciones llegaron por parte de las DDFF de Gipuzkoa y Bizkaia. El representante del Gobierno Vasco acuerda: comprobar y modificar el inventario de residuos: crear grupos de trabajo técnico, y analizar y en su caso modificar el documento de resolución a partir de la alegaciones expuestas y aportadas por escrito.

${ }^{15}$ El CVFP se recoge en el artículo 7 de la Ley 6/1989, de 6 de julio, de Función Pública Vasca.

${ }^{16}$ En la Ley 19/1997, de modificación de la Ley de Función Pública Vasca.

${ }^{17}$ La Diputación Foral de Araba votó en contra del Proyecto de decreto de modificación del decreto de indemnizaciones por razón de servicio. La Diputación Foral de Gipuzkoa votó en contra del Anteproyecto de modificación de la Ley de Función Pública Vasca. Por último, la Diputación Foral de Bizkaia y la Diputación Foral de Araba (no se encontraba presente la Diputación Foral de Gipuzkoa) votaron en contra del Proyecto de decreto de retribuciones de los/as fun. cionarios/as de las Administraciones Públicas Vascas para 1998.

\section{Bibliografía}

Álvarez Rico, M. (1982), «El principio de coordinación en el Estado de las Autonomías», en Documentación Administrativa, No. 196, Madrid: INAP: 5.48.

Agranoff, R. y Bañón, R. (1998), El Estado de las Autonomias: Hacia un nuevo federalismo?, Oñati: IVAP.

Calonge, A.; Garcia, J. A., y González, T. (1995), Autonomías y Municipios. Descentralización y coordinación de competencias, Valladolid: Secretariado de Publicaciones, Universidad de Valladolid.

Jiménez-Blanco, A. (1994), «Convenios de colaboración entre el Estado y las Comunidades Autónomas», en Documentación Administrativa, No. 240, Madrid: INAP: 93-107.

LASAGABASTER HeRrarTe, I. (1995), «Relaciones Intergubernamentales y Federalismo Cooperativo», en Revista Vasca de Administración Pública, No. 41, Onati: IVAP, 203.220.
MENÉNDEZ, P. (1993), Las potestades administrativas de dirección y de coordinación territorial, Madrid: Civitas.

MEnÉndeZ, A. (1994), «La cooperación, cun concepto jurídico?», en Documentación Administrativa, No. 240, Madrid: INAP: 11-51.

MorawinTz, R. (1985), La colaboración entre el Estado y los Entes Autonómicos territoriales en la Comunidad Europea, Madrid: Civitas.

Morell OcaNa, L. (1994), «Una teoría de la cooperación», en Documentación Administrativa, No. 240, Madrid: INAP: 51-71.

Rivero YseRn, J. L. (1994), «La cooperación provincial para la realización de servicios municipales», Documentación Administrativa, No. 240, Madrid: INAP: $131-165$.

Tornos Más, J. (1994), «Las Conferencias sectoriales», en Documentación Administrativa, No. 240, Madrid: INAP: 71-93. 


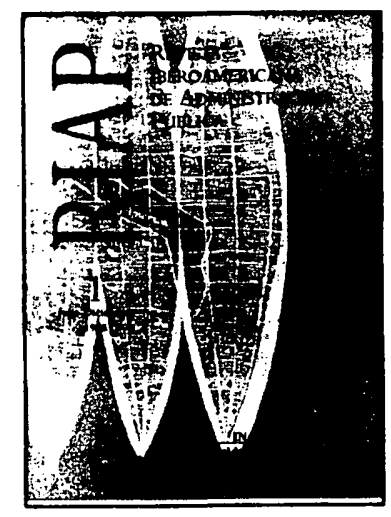

La REVISTA IBEROAmericana de ADMINISTRACión PúBLICA, RIAP, eś una nueva publicación semestral del Instituto Nacional de Administración Pública de España a través de la cual el INAP, continuando su tradición de acercamiento y colaboración con los países iberoamericanos, pretende crear nuevos vínculos de relación y cooperación entre las Administraciones Públicas de España e Iberoamérica.

Concebida como un foro abierto para la cooperación y el intercambio de información, opiniones y experiencias en el ámbito Iberoamericano de la Gestión Pública y las Ciencias de la Administración, la Revista Iberoamericana de Administración Pública, cuenta con un Consejor Rector Institucional presidido por el Ministro de Administraciones Públicas de España y con un Comité Científico integrado por destacados profesores e investigadores universitarios de Iberoamérica y de España.

La nueva Revista cuenta con un Índice estructurado en torno a cuatro Secciones: Artículos, Experiencias, Noticias y Documentos, dentro de los cuales tienen cabida desde las reflexiones doctrinales, hasta las crónicas de actualidad, el intercambio de informaciones sobre experiencias y actividades y la publicación de dossieres y documentos de interés mutuo.

Con todo ello, y mediante esta nueva publicación, el INAP de España desea continuar sus líneas de actuación respecto de Iberoamérica en las que a su misión institucional se le unen su vocación y su compromiso de presencia y colaboración con sus Administraciones Públicas.

\section{Director}

D. Enrique Álvarez Conde

\section{Secretario}

D. Jorge Urosa Sánchez

Coordinación

Centro de Publicaciones del INAP

Edita:

MINISTERIO DE ADMINISTRACIONES PÚBLICAS

Instituto Nacional de Administración Pública

\section{Suscripciones y Administración:}

Instituto Nacional de Administración Pública

Centro de Publicaciones

Atocha, 106. 28012 Madrid

Teléfono: $913493115-3235$

Fax: 913493287

E-mail: publicaciones@inap.map.es 